\title{
A challenging case of candida parapsilosis keratitis
}

\begin{abstract}
Purpose: To report challenges in the management of a patient with keratitis caused by Candida parapsilosis.

Methods: An otherwise healthy 48-year-old man developed chronic corneal ulcer following ocular trauma. Microbiologic work up using smear, culture and In Vivo Confocal Microscopy, was negative. Lamellar and penetrating keratoplasty were performed to cure the infection.
\end{abstract}

Results: The patient developed recurrence of the infection after lamellar keratoplasty. The interface recurrence was seen twice despite washing with antibiotics. Microbial culture finally revealed infection of $C$. parapsilosis which was sensitive to fluconazole and amphotericin B. Moreover, a repeat lamellar keratoplasty was followed by another recurrence. A penetrating keratoplasty was finally performed with no recurrence of infection during 10 months of follow-up.

Conclusions: Diagnosis of the corneal infection by C. parapsilosis can be challenging due to recurrence of the infection after lamellar keratoplasty. A penetrating keratoplasty may be required for cure of yeast infection after repeated recurrence of the infection. In addition, yeast infection should be considered in patients with chronic keratitis.

Keywords: Cornea - Keratitis - Candida parapsilosis - Keratoplasty

Submitted Date: 27 June 2018; Accepted Date: 04 July 2018; Published Date: 06 July 2018

\section{Introduction}

The emergence of various fungi which cause human infection has become an increasing serious public health problem worldwide. Fungal ocular infections, such as keratitis and endophthalmitis, may cause devasting vision loss. Fungal infection of the cornea accounts for up to $53 \%$ of all cases of infectious keratitis [1]. Many different species of fungi can infect the cornea depending on the region and climate. candida, fusarium, and aspergillus species are the most frequent fungal organisms causing keratitis. Yeasts, for example candida, are more frequently opportunistic than filamentous fungi for infection of cornea [2]. Yeast infections generally occur in compromised corneas with multiple predisposing alterations in the host defense, whereas filamentous fungal infections often occur in corneas with trauma caused by plants or vegetable matters $[3,4]$.

Most of the reported cases of yeast keratitis are due to candida species [3,5]. At least 17 species of candida are known to cause diseases in humans now [6]. For example, C. parapsilosis is the second or third most common cause of candidiasis, accounting for approximately $10 \%$ of all causes of yeast keratitis [7]. C. parapsilosis is commonly seen in the southern parts of the world and in Asia [8]. It is an ubiquitous microorganism in the natural environment, not only isolated easily from soil, seawater, and plants, but also can be isolated from mucosal surfaces, skin and nails [9-12]. Moreover, candida keratitis, which was first characterized in the middle of last century [13], is one of the most common fungi keratitis on normal human external eye [12]. Experimental studies have shown that
Ping Guo ${ }^{*}$, Jiaming Wang1, Hongbin Xie', Baotao Lin', Ming Li', Zhongliang Zou', Yuan Zhang ${ }^{2}$, Yingting Zhu ${ }^{2}$ and Ahmad Kheirkhah ${ }^{3}$

'Shenzhen Eye Hospital, School of Optometry and Ophthalmology of Shenzhen University, Shenzhen Key Laboratory of Ophthalmology, Shenzhen, China.

2Department of Research and Development, Tissue Tech, Inc, Miami, $\mathrm{FL}$, USA.

${ }^{3}$ Department of Ophthalmology, University of Texas Health Science Center at San Antonio, San Antonio, TX, USA.

*Author for correspondence:

E-mail: 2607212858@qq.com 
C. parapsilosis is generally less virulent than C. albicans or C. tropicalis [12]. However, an aggressive course of keratitis may be seen clinically in some cases.

The purpose of this report is to describe a severe case of $C$. parapsilosis keratitis with an aggressive course which reqired multiple therapeutic corneal grafts. A penetrating keratoplasty is highly recommended for cure of such yeast infection after repeated recurrence.

\section{Case and Methods}

A 48-year-old Chinese man presented to our hospital with a complaint of decreased vision in the left eye for 3 months. He had a history of corneal trauma to the left eye for which he had received corneal foreign body removal and antiinflammatory treatment in other hospitals.

Best corrected visual acuity was 20/20 in the right eye and 20/400 in the left eye. Slit lamp examination showed a central corneal ulcer measuring $7 \times 8 \mathrm{~mm}$ (Figure 1A). Anterior Segment Optical Coherence Tomography (AS-OCT) showed involvement of the deep corneal stroma. No hypha was found in In Vivo Confocal Microscopy, smear of corneal scrapings, or fungal culture. Bacterial culture was also negative. After a few days of antiinflammatory treatments, deep lamellar keratoplasty (LKP) was performed on his left eye. Fungus was also negative in pathogical section and culture in diseased cornea. Therefore one week postoperatively, he received topical treatment with tobramycin/ dexamethasone eyedrops and ointment. Three weeks after LKP, a small peripheral infiltration was noted at 9 o'clock position at the interface between the graft and the recipient bed (Figures 1B). We washed the interface between the cornea graft and the bed with tobramycin, and scraped for fungal and bacterial cultures. After the wash, he continuously received topical treatment with tobramycin/dexamethasone eyedrops and ointment. The microbial cultures were negative for both fungi and bacteria. Seven days later, the whitish infiltrate developed in the corneal graft again (Figure 1C). In Vivo Confocal Microscopy showed that collections of very small hypereflective structures in the graft, without presence of any filamentous fungal elements. AS-OCT showed the lesion at the interface. The interface between the cornea graft and the bed was thus washed again using vancomycin. We also scraped the bed for fungal and bacterial culture during the wash. Five days after the wash, white colonies with clear boundaries were noted on the graft (Figure 1D). AS-OCT showed corneal graft edema and In Vivo Confocal Microscopy demonstrated edema and opacity with dense highly reflective structures in the stroma of the cornea graft (Figure 1E). The culture from interface specimen showed Candida parapsilosis (Figures $1 \mathrm{~F}$ and $1 \mathrm{G}$ ) which was sensitive to both fluconazole and amphotericin B. We stopped using tobramycin/ dexamethasone eyedrops and ointment immediately and treated the patient with oral fluconazole and topical fluconazole combined with amphotericin B. Then, we performed the third interlayer wash using fluconazole. The corneal bed was clear, transparent, and no infiltration was seen. The corneal graft, however, was not cleaned and obvious opacity could be found. After sufficient communication with patients, we performed LKP during the surgery using a fresh donor cornea. Three weeks after LKP, fungal recurrence occurred along with interlayer empyema (Figures $2 \mathrm{~A}$ and $2 \mathrm{~B}$ ). The patient received the third-time operation and this time he received penetrating keratoplasty. Two months postoperatively, the corneal graft was transparent and his BCVA was 20/100 (Figure 3A). During a follow-up of 10 months, the patient did not show any recurrence of fungal infection in the cornea graft (Figure 3B).
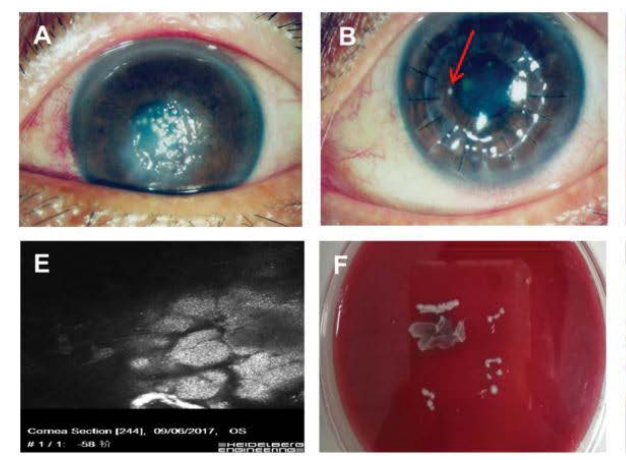
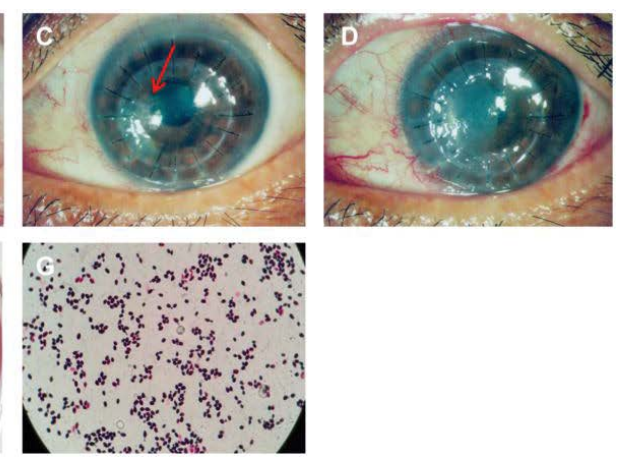

Figure 1: Clinical and laboratory manisfications of Candida Parapsilosis Keratitis. (A) Central corneal ulcer measuring $7 \times 8 \mathrm{~mm}$. B. Peripheral infiltration interlamellar 3 weeks after LKP. C. Whitish infiltrate was present 7 days after first flush. D. Clear white colonies interlamellar 5 days after second flush. E. Colonies was seen in vivo confocal microscopy. F and G. Candida parapsilosis was confirmed in the culture from interface specimen. 

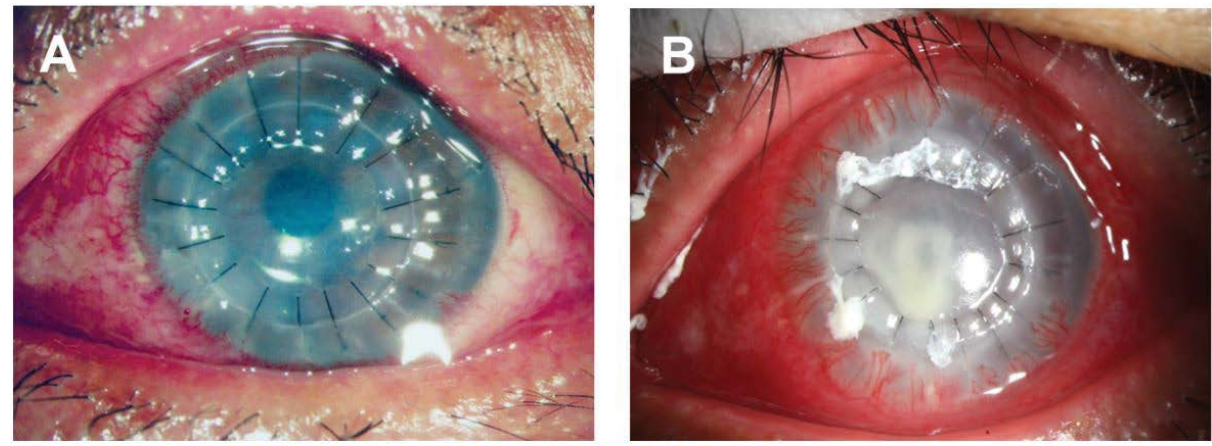

Figure 2: Clinical appearance of Candida Parapsilosis Keratitis after LKP. (A) 1 day after LKP. Figure 2B. 21 days after LKP.
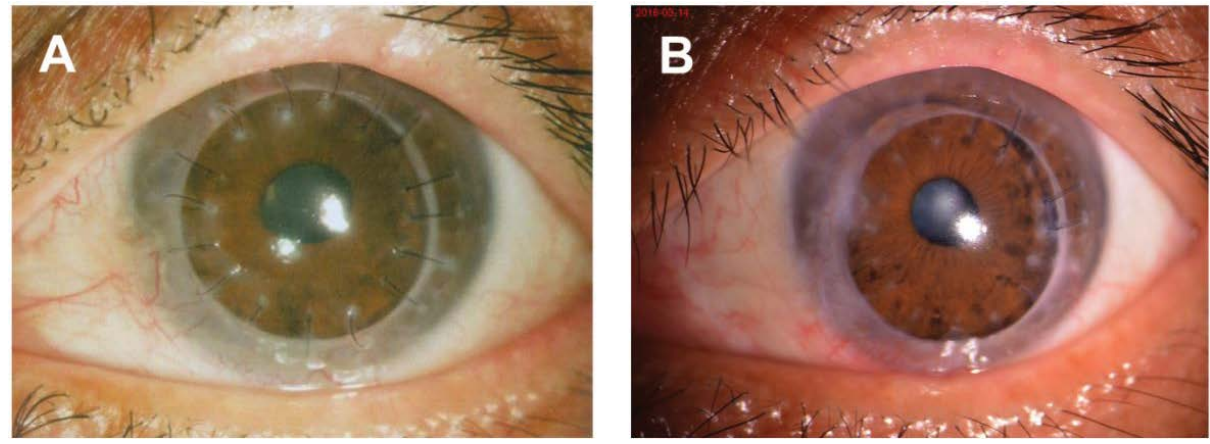

Figure 3: Clinical appearance of Candida Parapsilosis Keratitis after PKP. (A) 2 months after PKP. Figure 10 months after PKP.

\section{Discussion}

The vast majority of fungal corneal infections are caused by filamentous fungi [14]. However, compromised corneas are prone to yeast infections, usually by candida species. Corneal surgical procedures such as corneal transplantation and refractive surgery, steroid eyedrops, ocular trauma, and ocular surface disease are the major risk factors [15-18]. More frequently opportunistic $C$. parapsilosis has recently been found with increasing frequency. However, rapid diagnosis of $C$. parapsilosis keratitis can be challenging. First, traditional inspection methods, such as corneal smear and confocal exams, and pathological exams were used to looking for mycelium in the pathological tissue. However, lack of typical fungal mycelium in $C$. parapsilosis keratitis made this diagnosis more difficult. Besides, clinical presentation of $C$. parapsilosis keratitis shows a great diversity. Information on the antimicrobial susceptibility of $C$. parapsilosis is limited by lack of standardized methods. Furthermore, topical steroid eyedrops were usually used post corneal transplantation if fungal infection was not confirmed. As a consequence, prognosis of the ocular infection can be very poor and this can lead to devasting vision loss in C. parapsilosis keratitis. Therefore, it is very important to make a correct diagnois of the corneal infection by yeasts using repeated corneal scraping, tissue culture, In
Vivo Confocal microscopy, and pathological exams. The treatment of the corneal infection by $C$. parapsilosis is challenging because of recurrence of the infection after lamellar keratoplasty. For example, our patient reported here developed repeated recurrence of the infection of parapsilosis after lamellar keratoplasty confirmed by various examinations (Figure 1). Such repeated infections were not cured by LKP (Figure 2) but were finally cured by a penetrating keratoplasty (Figure 3), suggesting that a penetrating keratoplasty is effective for treatment of repeated yeast infection.

\section{Conclusion}

In conclusion, this case demonstrates that prognosis of $C$. parapsilosis keratitis can be poor and emphasizes the importance of microbiologic examination of any infectious keratitis. In addition, a penetrating keratoplasty is necessary for effective treatment of repeated recurrence of yeast infection.

\section{Conflict of Interest Declaration}

No conflict of interest is declared from all authors.

\section{Acknowledgment}

This work was supported by Grant 2015A030313774, Natural Science Fund of Guangdong Province, China. 
Executive summary

Purpose: To report challenges in the management of a patient with keratitis caused by Candida parapsilosis.

Methods: An otherwise healthy 48-year-old man developed chronic corneal ulcer following ocular trauma. Microbiologic work up using smear, culture and In Vivo Confocal Microscopy, was negative. Lamellar and penetrating keratoplasty were performed to cure the infection.

Results: The patient developed recurrence of the infection after lamellar keratoplasty. The interface recurrence was seen twice despite washing with antibiotics. Microbial culture finally revealed infection of $C$. parapsilosis which was sensitive to fluconazole and amphotericin B. Moreover, a repeat lamellar keratoplasty was followed by another recurrence. A penetrating keratoplasty was finally performed with no recurrence of infection during 10 months of follow-up.

Conclusions: Diagnosis of the corneal infection by C. parapsilosis can be challenging due to recurrence of the infection after lamellar keratoplasty. A penetrating keratoplasty may be required for cure of yeast infection after repeated recurrence of the infection. In addition, yeast infection should be considered in patients with chronic keratitis.

\section{References}

1. Alkatan HM, Maktabi A, Al-Harby MAl-Rajhi AA. Candida parapsilosis corneal graft infection from a single eye center: Histopathologic report of 2 cases. Saudi J Ophthalmol 29: 303306 (2015).

2. Mascarenhas J, Lalitha P, Prajna NV, Srinivasan M, Das M, D'Silva SS, Oldenburg CE, Borkar DS, Esterberg EJ, Lietman TMKeenan JD. Acanthamoeba, fungal, and bacterial keratitis: a comparison of risk factors and clinical features. Am J Ophthalmol 157:56-62 (2014)

3. Tanure MA, Cohen EJ, Sudesh S, Rapuano CJLaibson PR. Spectrum of fungal keratitis at Wills Eye Hospital, Philadelphia, Pennsylvania. Cornea 19: 307-312 (2000).

4. Rosa RH Jr., Miller DAlfonso EC. The changing spectrum of fungal keratitis in south Florida. Ophthalmol 101: 1005-1013 (1994).

5. Wilhelmus KRRobinson NM. Infectious crystalline keratopathy caused by Candida albicans. Am J Ophthalmol 112: 322-325 (1991).

6. Lalla RV, Patton LLDongari-Bagtzoglou A. Oral candidiasis: pathogenesis, clinical presentation, diagnosis and treatment strategies. J Calif Dent Assoc 41: 263-268 (2013).

7. van Asbeck EC, Clemons KVStevens DA. Candida parapsilosis: a review of its epidemiology, pathogenesis, clinical aspects, typing and antimicrobial susceptibility. Crit Rev Microbiol 35: 283-309 (2009).

8. Arendrup MC. Epidemiology of invasive candidiasis. Curr Opin Crit Care 16: 445-452 (2010).

9. De Bernardis F, Mondello F, San Millan R, Ponton JCassone A. Biotyping and virulence properties of skin isolates of Candida parapsilosis. J Clin Microbiol 37: 3481-3486 (1999).
10. Kuhn DM, Mikherjee PK, Clark TA, Pujol C, Chandra J, Hajjeh RA, Warnock DW, Soil DRGhannoum MA. Candida parapsilosis characterization in an outbreak setting. Emerg Infect Dis 10: 1074-1081 (2004).

11. Sanchez V, Vazquez JA, Barth-Jones D, Dembry L, Sobel JDZervos MJ. Nosocomial acquisition of Candida parapsilosis: an epidemiologic study. Am J Med 94: 577-582 (1993).

12. Weems JJ, Jr. Candida parapsilosis: epidemiology, pathogenicity, clinical manifestations, and antimicrobial susceptibility. Clin Infect Dis 14: 756-766 (1992).

13. Sykes EM. Fungus infections of the cornea; case report of keratomycosis due to monilia. Tex State J Med 42: 330-332 (1946).

14. Chirinos-Saldana P, Bautista de Lucio VM, Hernandez-Camarena JC, Navas A, Ramirez-Miranda A, Vizuet-Garcia L, Ortiz-Casas M, Lopez-Espinosa N, Gaona-Juarez C, Bautista-Hernandez LAGraue-Hernandez EO. Clinical and microbiological profile of infectious keratitis in children. BMC Ophthalmol 13: 54 (2013).

15. Harris DJ, Jr., Stulting RD, Waring GO, 3rdWilson LA. Late bacterial and fungal keratitis after corneal transplantation. Spectrum of pathogens, graft survival, and visual prognosis. Ophthalmol 95: 1450-1457 (1988).

16. Tseng SHLing KC. Late microbial keratitis after corneal transplantation. Cornea 14: $591-594$ (1995).

17. Matsumoto A, Sano Y, Nishida K, Yokoi N, Hara JKinoshita S. A case of infectious crystalline keratopathy occurring long after penetrating keratoplasty. Cornea 17: 119-122 (1998).

18. Akova YA, Onat M, Koc F, Nurozler ADuman S. Microbial keratitis following penetrating keratoplasty. Ophthalmic Surg Lasers 30: 449-455 (1999). 